\title{
Vegetarian diet and cholesterol and TAG levels by gender
}

\author{
Zhi-Hong Jian ${ }^{1}$, Yi-Chen Chiang ${ }^{1}$, Chia-Chi Lung ${ }^{1}$, Chien-Chang Ho', Pei-Chieh Ko', \\ Oswald Ndi Nfor ${ }^{1}$, Hui-Chin Chang ${ }^{1,3}$, Yi-Ching Liaw ${ }^{1,3}$, Yu-Chiu Liang ${ }^{4}$ and \\ Yung-Po Liaw ${ }^{1,5, *}$ \\ 'Department of Public Health and Institute of Public Health, Chung Shan Medical University, No. 110 Sec. 1 Jianguo N. \\ Road, Taichung City 40201, Taiwan, Republic of China: ${ }^{2}$ Department of Health and Leisure Management, Yuanpei \\ University, Hsinchu City, Taiwan, Republic of China: ${ }^{3}$ EBM Center \& Library, Chung Shan Medical University \\ Hospital, Taichung City, Taiwan, Republic of China: ${ }^{4}$ Department of Exercise and Health Promotion, Ta Hwa \\ University of Science and Technology, Hsinchu County, Taiwan, Republic of China: ${ }^{5}$ Department of Family and \\ Community Medicine, Chung Shan Medical University Hospital, Taichung City, Taiwan, Republic of China
}

Submitted 19 December 2013: Final revision received 27 March 2014: Accepted 3 April 2014: First published online 25 June 2014

\begin{abstract}
Objective: The present study assessed the effects of vegetarian and omnivorous diets on HDL-cholesterol (HDL-C), LDL-cholesterol (LDL-C), TAG and the ratio of HDL-C to total cholesterol (TC) by gender.

Design: HDL-C, LDL-C, TAG and HDL-C:TC were compared among three diet groups (vegan, ovo-lacto vegetarian and omnivorous). Multivariate linear regression analysis was performed to examine factors significantly and independently associated with vegetarian status and to estimate the $\beta$ value of lipid profiles for the diet groups.

Settings: A cross-sectional study. Data were obtained from the Taiwanese Survey on the Prevalence of Hyperglycemia, Hyperlipidemia and Hypertension (TwSHHH).

Subjects: The study comprised included 3257 men and 3551 women.

Results: After adjusting for confounders, vegan and ovo-lacto vegetarian diets lowered LDL-C levels $(\beta=-10 \cdot 98, P=0.005$ and $\beta=-7 \cdot 12, P=0 \cdot 025$, respectively) in men compared with omnivorous diet. There was a significant association between HDL-C and vegan diet $(\beta=-6.53, P=0.004)$. In females, the $\beta$ values of HDL-C, TAG and HDL-C:TC were $-5.72(P<0.0001), 16.51(P=0.011)$ and -0.02 $(P=0.012)$ for vegan diet, and $-4.86(P=0.002), 15.09(P=0.008)$ and -0.01 $(P=0.026)$ for ovo-lacto vegetarian diet, when compared with omnivorous diet. Conclusions: Vegan diet was associated with lower HDL-C concentrations in both males and females. Because the ovo-lacto vegetarian diet was effective in lowering LDL-C, it may be more appropriate for males.
\end{abstract}

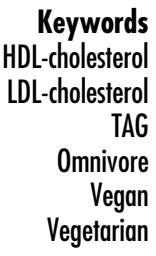

Vegans and ovo-lacto vegetarians are the two common varieties of vegetarians. Vegetarians consume smaller amounts of total fat and saturated fat and larger amounts of unsaturated fats and fibre than omnivores ${ }^{(1)}$. The consumption of a vegetarian diet is believed to reduce the risk of $\mathrm{CVD}^{(2-5)}$. HDL-cholesterol (HDL-C), LDL-cholesterol (LDL-C), TAG and the ratio of HDL-C to total cholesterol (TC) are often used to predict the risk of having CVD $^{(6,7)}$. Better plasma lipid and lipoprotein profiles are proven strategies to reduce risk for $\mathrm{CVD}^{(7)}$.

Vegetarians tend to have lower LDL-C and TC than omnivorous individuals $^{(8)}$. Despite having lower HDL-C and LDL-C levels, the ratio TC:HDL-C ratio was lower in female vegetarians $^{(9)}$. Healthy postmenopausal vegetarians had lower TC, LDL-C and $\mathrm{TAG}^{(10)}$. Fu et al. also reported that postmenopausal vegetarians had lower TC, HDL-C and LDL-C than age-matched omnivores. In a meta-analysis, vegetarian diets were effective in lowering plasma TAG concentrations, especially in developing countries ${ }^{(11)}$. HDL-C is an independent risk factor of $\mathrm{CVD}^{(12)}$. In our previous work, coronary artery disease risk was higher in patients with low levels of HDL-C than in those with high levels of LDL-C ${ }^{(13)}$. Results from previous studies on the effects of vegetarian diets on lipids, especially HDL-C, have been inconsistent. Besides, CVD is relatively more common in postmenopausal women compared with age-matched men or premenopausal women ${ }^{(14)}$. In this regard, the present study focused on the effects of vegetarian diets on HDL-C, LDL-C, TAG and HDL-C:TC by gender. 


\section{Materials and methods}

The data were obtained from the 2002 Taiwanese Survey on the Prevalence of Hyperglycemia, Hyperlipidemia and Hypertension (TwSHHH) for individuals aged 15 years and above. The survey was approved, conducted and provided by the National Health Research Institutes and Bureau of Health Promotion. Information was collected on age, gender, exercise, dietary habits and organic food. Measurements included waist:hip ratio (WHR), blood pressure and serum biochemistry. Individuals who had received lipid-lowering drugs were excluded. Participants were instructed to fast for more than $12 \mathrm{~h}$ prior to blood sampling. Blood samples were collected to determine blood glucose, HDL-C, LDL-C, apo-A1, apo-B, TAG, TC, uric acid and creatinine.

Vegetarians were identified from the responses to the interview questions. The questions were related to dietary type (vegan and ovo-lacto vegetarian). The questionnaires did not contain the exact amount of diet and the recommended daily intake. Vegan diet was defined as a diet that excluded eggs, milk, meat, poultry, seafood and byproducts of animals, while ovo-lacto vegetarian diet was one that excluded all meat and fish but included eggs and dairy products, for a period of 1 year.

Confounders included gender, age, exercise, organic food, WHR, blood pressure, fasting plasma glucose, uric acid and creatinine. Two seated blood pressure readings were taken $10 \mathrm{~s}$ apart in the left arm after a 5-10 min rest. Another blood pressure measurement was taken if the first two readings varied by more than $10 \mathrm{mmHg}$. The mean of the two closest readings was calculated. Hip circumference was measured at the level of the greater trochanter. Waist circumference was measured midway between the iliac crest and the last rib. WHR was calculated as waist circumference (centimetres) divided by hip circumference (centimetres) as the index of central obesity. Because body weights were not measured during the survey, obesity was defined as WHR $\geq 0.90$ for men and $\geq 0.85$ for women ${ }^{(15)}$. Exercise was defined by individuals who had regular exercise at least three times per week (30 min each time) for 3 months or more. An organic food diet was defined as a diet in which half of the food was from an organic grocery store and for consumers who had followed such a diet for at least 1 year prior to the study.

\section{Statistical analysis}

All analyses were conducted by using the SAS statistical software package version 9.2. Continuous data were expressed as mean and standard deviation. Comparisons among vegans, ovo-lacto vegetarians and omnivores were made using ANOVA. Multiple linear regression analysis was used to estimate the association between serum lipid levels, associated factors and the three diet groups. A $P$ value $<0.05$ was considered to be statistically significant.

\section{Results}

The study included 3257 men (forty-five vegans, sixtyeight ovo-lacto vegetarians and 3144 omnivores) and 3551 women (ninety-nine vegans, 127 lacto-ovo vegetarians and 3325 omnivores). The demographic, laboratory and anthropometric characteristics of males are shown in Table 1. There were no significant differences in waist circumference, WHR, blood pressure, fasting plasma glucose, apo-A1, apo-B, TAG and HDL-C:TC among the three

Table 1 Demographic, laboratory and anthropometric characteristics of vegan, ovo-lacto vegetarian and omnivorous men, Taiwanese Survey on the Prevalence of Hyperglycemia, Hyperlipidemia and Hypertension (TwSHHH), 2002

\begin{tabular}{|c|c|c|c|c|c|c|c|}
\hline \multirow[b]{2}{*}{ Variable } & \multicolumn{2}{|c|}{$\begin{array}{l}\text { Vegans } \\
(n 45)\end{array}$} & \multicolumn{2}{|c|}{$\begin{array}{l}\text { Ovo-lacto vegetarians } \\
\qquad(n 68)\end{array}$} & \multicolumn{2}{|c|}{$\begin{array}{l}\text { Omnivores } \\
(n 3144)\end{array}$} & \multirow[b]{2}{*}{$P$ value } \\
\hline & Mean & SD & Mean & SD & Mean & SD & \\
\hline Age (years) & $51 \cdot 5^{\star}$ & $16 \cdot 2$ & $45 \cdot 5$ & $15 \cdot 6$ & $43 \cdot 3$ & $17 \cdot 5$ & 0.005 \\
\hline WC (cm) & $81 \cdot 7$ & 8.7 & $82 \cdot 4$ & $10 \cdot 3$ & $84 \cdot 2$ & $10 \cdot 7$ & 0.111 \\
\hline $\mathrm{HC}(\mathrm{cm})$ & $94 \cdot 2^{\star}$ & 6.5 & 95.4 & $8 \cdot 2$ & $97 \cdot 0$ & $7 \cdot 8$ & 0.013 \\
\hline WHR & 0.87 & 0.06 & 0.86 & 0.07 & 0.87 & 0.08 & 0.913 \\
\hline SBP (mmHg) & 121.9 & $20 \cdot 0$ & $117 \cdot 0$ & $17 \cdot 5$ & $118 \cdot 9$ & $16 \cdot 3$ & 0.287 \\
\hline DBP (mmHg) & 78.4 & $10 \cdot 1$ & $76 \cdot 4$ & $11 \cdot 0$ & $77 \cdot 7$ & $10 \cdot 9$ & 0.569 \\
\hline FPG (mg/dl) & 92.9 & $13 \cdot 1$ & $98 \cdot 4$ & $38 \cdot 8$ & $94 \cdot 7$ & $27 \cdot 8$ & 0.494 \\
\hline UA (mg/dl) & 6.9 & 1.8 & $6 \cdot 8$ & 1.5 & $7 \cdot 2$ & 1.7 & 0.059 \\
\hline Creatinine (mg/dl) & 1.0 & 0.2 & 1.0 & 0.2 & 1.0 & 0.3 & 0.336 \\
\hline HDL-C (mg/dl) & $45 \cdot 8^{\star}$ & $15 \cdot 0$ & $50 \cdot 5$ & $14 \cdot 3$ & $51 \cdot 6$ & 14.5 & 0.030 \\
\hline LDL-C (mg/dl) & $104 \cdot 6^{\star}$ & $25 \cdot 8$ & $108 \cdot 3^{*}$ & $24 \cdot 8$ & 116.4 & $26 \cdot 7$ & 0.001 \\
\hline Apo-A1 (mg/dl) & 134.5 & $17 \cdot 7$ & 130.9 & $20 \cdot 0$ & $137 \cdot 1$ & 23.0 & 0.083 \\
\hline Apo-B (mg/dl) & 83.6 & $22 \cdot 3$ & 88.0 & $24 \cdot 2$ & 91.6 & $25 \cdot 0$ & 0.066 \\
\hline TAG (mg/dl) & $156 \cdot 5$ & $104 \cdot 9$ & $136 \cdot 8$ & 71.0 & 141.6 & $95 \cdot 1$ & 0.548 \\
\hline $\mathrm{TC}(\mathrm{mg} / \mathrm{dl})$ & $164 \cdot 2^{\star}$ & $36 \cdot 1$ & $172 \cdot 4$ & 33.2 & $181 \cdot 7$ & 35.9 & 0.011 \\
\hline HDL-C:TC & 0.28 & 0.08 & 0.30 & 0.08 & 0.29 & 0.08 & 0.598 \\
\hline
\end{tabular}

WC, waist circumference; HC, hip circumference; WHR, waist:hip ratio; SBP, systolic blood pressure; DBP, diastolic blood pressure; FPG, fasting plasma glucose; UA, uric acid; HDL-C, HDL-cholesterol; LDL-C, LDL-cholesterol; TC; total cholesterol.

${ }^{\star}$ Mean values were significantly different from those of omnivores $(P<0.05)$. 
diet groups. Compared with omnivores, vegans were significantly older and had lower levels of HDL-C, LDL-C and TC.

Table 2 shows the demographic, laboratory and anthropometric characteristics of female vegans, ova-lacto vegetarians and omnivores. There were no significant differences between diet groups for waist circumference, hip circumference, fasting plasma glucose, LDL-C and TC. Vegans registered lowest HDL-C:TC and highest age, WHR and TAG levels, which were significantly different compared with omnivores. HDL-C was significantly lower in vegans and ovo-lacto vegetarians than in omnivores.
The associations between lipid profiles and dietary groups for males assessed by multiple linear regression analyses are shown in Table 3. After adjusting for potential confounders, vegan and ovo-lacto vegetarian diets had significant effects on LDL-C $(\beta=-10 \cdot 98, P=0.005$ and $\beta=-7 \cdot 12, P=0 \cdot 025$, respectively). Significant associations were observed between vegan diet and HDL-C $(\beta=-6.53$, $P=0.004)$ compared with omnivorous diet. Exercise was associated with higher HDL-C $(\beta=1 \cdot 16, P=0 \cdot 045)$ and HDL-C:TC $(\beta=0 \cdot 01, P=0 \cdot 001)$ and lower TAG levels $(\beta=-7 \cdot 91, P=0 \cdot 026)$. There was no significant association between organic foods and lipid profiles.

Table 2 Demographic, laboratory and anthropometric characteristics of vegan, ovo-lacto vegetarian and omnivorous women, Taiwanese Survey on the Prevalence of Hyperglycemia, Hyperlipidemia and Hypertension (TwSHHH), 2002

\begin{tabular}{|c|c|c|c|c|c|c|c|}
\hline \multirow[b]{2}{*}{ Variable } & \multicolumn{2}{|c|}{$\begin{array}{l}\text { Vegans } \\
(n 99)\end{array}$} & \multicolumn{2}{|c|}{$\begin{array}{l}\text { Ovo-lacto vegetarians } \\
\text { ( } n \text { 127) }\end{array}$} & \multicolumn{2}{|c|}{$\begin{array}{l}\text { Omnivores } \\
(n \text { 3325) }\end{array}$} & \multirow[b]{2}{*}{$P$ value } \\
\hline & Mean & SD & Mean & SD & Mean & SD & \\
\hline Age (years) & $54 \cdot 4^{*}, \dagger$ & 13.6 & $47 \cdot 7^{*}$ & $15 \cdot 3$ & $42 \cdot 8$ & $16 \cdot 7$ & $<0.0001$ \\
\hline WC $(\mathrm{cm})$ & $77 \cdot 2$ & $10 \cdot 2$ & $76 \cdot 1$ & 9.9 & 75.6 & 10.5 & 0.288 \\
\hline $\mathrm{HC}(\mathrm{cm})$ & 94.9 & $9 \cdot 1$ & 97.0 & 8.7 & $96 \cdot 0$ & 8.3 & 0.182 \\
\hline WHR & $0.81^{\star}, \dagger$ & 0.07 & 0.78 & 0.06 & 0.79 & 0.07 & 0.001 \\
\hline $\mathrm{SBP}(\mathrm{mmHg})$ & $119 \cdot 0^{*}$ & $20 \cdot 3$ & 114.6 & $17 \cdot 8$ & $112 \cdot 2$ & 18.9 & 0.001 \\
\hline $\mathrm{DBP}(\mathrm{mmHg})$ & 74.4 & $10 \cdot 7$ & 71.9 & $10 \cdot 8$ & $72 \cdot 2$ & $10 \cdot 9$ & 0.172 \\
\hline FPG $(\mathrm{mg} / \mathrm{dl})$ & 94.8 & $25 \cdot 3$ & 95.4 & 30.7 & $93 . \overline{7}$ & 28.0 & 0.750 \\
\hline UA (mg/dl) & 5.5 & 1.5 & $5 \cdot 3$ & 1.5 & $5 \cdot 6$ & 1.5 & 0.203 \\
\hline Creatinine (mg/dl) & 0.8 & 0.1 & 0.8 & 0.1 & 0.8 & 0.3 & 0.419 \\
\hline HDL-C (mg/dl) & $54.9^{*}$ & $12 \cdot 0$ & $55 \cdot 1^{*}$ & 13.8 & $59 \cdot 3$ & $14 \cdot 2$ & $<0.0001$ \\
\hline LDL-C (mg/dl) & $117 \cdot 7$ & 28.4 & 112.5 & 31.2 & 114.4 & $27 \cdot 1$ & 0.371 \\
\hline Apo-A1 (mg/dl) & $150 \cdot 9$ & $22 \cdot 0$ & 145.9 & 19.2 & 151.0 & $25 \cdot 1$ & 0.083 \\
\hline Apo-B (mg/dl) & $90 \cdot 3$ & 24.4 & 84.3 & $28 \cdot 1$ & $85 \cdot 7$ & 24.9 & 0.164 \\
\hline TAG (mg/dl) & $135.4^{*}$ & $66 \cdot 7$ & $126 \cdot 1$ & 77.4 & 111.0 & 70.9 & $<0.001$ \\
\hline $\mathrm{TC}(\mathrm{mg} / \mathrm{dl})$ & $184 \cdot 7$ & 35.2 & 178.5 & $43 \cdot 1$ & 184.3 & 37.4 & 0.245 \\
\hline HDL-C:TC & $0.30^{*}$ & 0.07 & 0.31 & 0.07 & 0.33 & 0.07 & 0.001 \\
\hline
\end{tabular}

WC, waist circumference; HC, hip circumference; WHR, waist:hip ratio; SBP, systolic blood pressure; DBP, diastolic blood pressure; FPG, fasting plasma glucose; UA, uric acid; HDL-C, HDL-cholesterol; LDL-C, LDL-cholesterol; TC; total cholesterol.

${ }^{*}$ Mean values were significantly different from those of omnivores $(P<0.05)$.

†Mean values were significantly different from those of ovo-lacto vegetarians $(P<0.05)$.

Table 3 Regression analysis between diet types and serum lipid levels in men, Taiwanese Survey on the Prevalence of Hyperglycemia, Hyperlipidemia and Hypertension (TwSHHH), 2002

\begin{tabular}{|c|c|c|c|c|c|c|c|c|}
\hline \multirow[b]{2}{*}{ Variable } & \multicolumn{2}{|c|}{ HDL-C } & \multicolumn{2}{|c|}{ LDL-C } & \multicolumn{2}{|c|}{ TAG } & \multicolumn{2}{|c|}{ HDL-C:TC } \\
\hline & $\beta$ & $P$ value & $\beta$ & $P$ value & $\beta$ & $P$ value & $\beta$ & $P$ value \\
\hline \multicolumn{9}{|l|}{ Diet } \\
\hline Omnivorous diet & - & - & - & - & - & - & - & - \\
\hline Ovo-lacto vegetarian diet & $-1 \cdot 76$ & 0.330 & $-7 \cdot 12$ & 0.025 & 1.83 & 0.868 & 0.004 & 0.700 \\
\hline Vegan diet & -6.53 & 0.004 & -10.98 & 0.005 & 21.23 & 0.121 & -0.01 & 0.350 \\
\hline Exercise & $1 \cdot 16$ & 0.045 & -1.69 & 0.097 & -7.91 & 0.026 & 0.01 & 0.001 \\
\hline Organic food & 0.55 & 0.860 & $-10 \cdot 32$ & 0.058 & -14.17 & 0.454 & 0.02 & 0.149 \\
\hline Obesity* & -4.64 & $<0.0001$ & 6.73 & $<0.0001$ & $42 \cdot 86$ & $<0.0001$ & -0.04 & $<0.0001$ \\
\hline \multicolumn{9}{|l|}{ Age (years) } \\
\hline$<45$ & - & - & - & - & - & - & - & - \\
\hline$\geq 45$ to $<60$ & $3 \cdot 30$ & $<0.0001$ & 9.50 & $<0.0001$ & 1.28 & 0.752 & -0.01 & $0 \cdot 131$ \\
\hline$\geq 60$ & 4.48 & $<0.0001$ & $6 \cdot 65$ & $<0.0001$ & $-22 \cdot 24$ & $<0.0001$ & 0.01 & 0.059 \\
\hline SBP & -0.05 & 0.054 & -0.02 & 0.617 & 0.17 & 0.274 & -0.0002 & 0.247 \\
\hline DBP & 0.04 & 0.292 & 0.30 & $<0.0001$ & 1.08 & $<0.0001$ & -0.001 & 0.002 \\
\hline FPG & -0.02 & 0.010 & 0.08 & $<0.0001$ & 0.53 & $<0.0001$ & -0.003 & $<0.0001$ \\
\hline UA & -0.42 & 0.010 & 1.76 & $<0.0001$ & 9.60 & $<0.0001$ & -0.006 & $<0.0001$ \\
\hline Creatinine & $2 \cdot 38$ & 0.011 & $6 \cdot 68$ & $<0.0001$ & -0.76 & 0.895 & -0.003 & 0.506 \\
\hline
\end{tabular}

HDL-C, HDL-cholesterol; LDL-C, LDL-cholesterol TC, total cholesterol; SBP, systolic blood pressure; DBP, diastolic blood pressure; FPG, fasting blood glucose; UA, uric acid.

${ }^{*}$ Obesity is defined as waist:hip ratio $\geq 0 \cdot 90$. 
Table 4 Regression analysis between diet types and serum lipid levels in women, Taiwanese Survey on the Prevalence of Hyperglycemia, Hyperlipidemia and Hypertension (TwSHHH), 2002

\begin{tabular}{|c|c|c|c|c|c|c|c|c|}
\hline \multirow[b]{2}{*}{ Variable } & \multicolumn{2}{|c|}{ HDL-C } & \multicolumn{2}{|c|}{ LDL-C } & \multicolumn{2}{|c|}{ TAG } & \multicolumn{2}{|c|}{ HDL-C:TC } \\
\hline & $\beta$ & $P$ value & $\beta$ & $P$ value & $\beta$ & $P$ value & $\beta$ & $P$ value \\
\hline \multicolumn{9}{|l|}{ Diet } \\
\hline Omnivorous diet & - & - & - & - & - & - & _- & - \\
\hline Ovo-lacto vegetarian diet & -4.86 & 0.002 & -3.15 & 0.159 & $15 \cdot 09$ & 0.008 & -0.01 & 0.026 \\
\hline Vegan diet & -5.72 & $<0.0001$ & -2.53 & 0.322 & $16 \cdot 51$ & 0.011 & -0.02 & 0.012 \\
\hline Menopause & $2 \cdot 60$ & 0.003 & 8.40 & $<0.0001$ & 6.07 & 0.118 & -0.01 & 0.109 \\
\hline Exercise & 1.07 & 0.073 & $-1 \cdot 10$ & 0.289 & -7.50 & 0.004 & 0.01 & 0.013 \\
\hline Organic food & -2.67 & 0.297 & -1.46 & 0.744 & 1.38 & 0.903 & -0.01 & 0.647 \\
\hline Obesity* & -4.00 & $<0.0001$ & -0.85 & 0.504 & 17.46 & $<0.0001$ & -0.02 & $<0.0001$ \\
\hline \multicolumn{9}{|l|}{ Age (years) } \\
\hline$<45$ & - & - & - & - & - & - & - & - \\
\hline$\geq 45$ to $<60$ & 4.63 & $<0.0001$ & $9 \cdot 12$ & $<0.0001$ & $9 \cdot 31$ & 0.004 & -0.003 & 0.312 \\
\hline$\geq 60$ & 2.69 & 0.020 & 6.91 & 0.001 & 14.51 & 0.005 & -0.01 & 0.223 \\
\hline SBP & -0.01 & 0.633 & 0.12 & 0.004 & -0.02 & 0.876 & -0.0002 & 0.044 \\
\hline DBP & -0.09 & 0.007 & 0.13 & 0.037 & 0.79 & $<0.0001$ & -0.001 & $<0.0001$ \\
\hline FPG & -0.02 & 0.029 & 0.14 & $<0.0001$ & 0.64 & $<0.0001$ & -0.0004 & $<0.0001$ \\
\hline UA & -0.52 & 0.002 & $2 \cdot 78$ & $<0.0001$ & $10 \cdot 27$ & $<0.0001$ & -0.01 & $<0.0001$ \\
\hline Creatinine & $1 \cdot 12$ & 0.144 & 0.50 & 0.706 & 0.22 & 0.949 & 0.004 & 0.265 \\
\hline
\end{tabular}

HDL-C, HDL-cholesterol; LDL-C, LDL-cholesterol TC, total cholesterol; SBP, systolic blood pressure; DBP, diastolic blood pressure; FPG, fasting blood glucose; UA, uric acid.

${ }^{*}$ Obesity is defined as waist:hip ratio $\geq 0.85$.

The associations between serum lipid levels and dietary groups for females generated by multiple linear regression analyses are shown in Table 4. Vegan and ovo-lacto vegetarian diets were significantly associated with lower HDL-C $(\beta=-5.72, P<0.0001$ and $\beta=-4.86, P=0.002$, respectively) and HDL-C:TC $(\beta=-0.02, P<0.012$ and $\beta=-0.01, \quad P=0.026$, respectively), and higher TAG $(\beta=16.51, \quad P=0.011$ and $\beta=15.09, \quad P=0.008$, respectively), compared with omnivorous diet. The difference associated with menopausal status was $2.60(P=0.003)$ for HDL-C and $8.40(P<0.0001)$ for LDL-C. Exercise was associated with a decrease in TAG level $(\beta=-7.50$, $P=0.004)$ and an increase in HDL-C:TC $(\beta=0.01$, $P=0 \cdot 013)$. No significant association was found between organic foods and lipid profiles.

\section{Discussion}

Lower levels of TAG, TC and LDL-C have been reported in vegans $^{(16)}$. However, to our knowledge, no nationwide study has assessed the health effects of different diet groups on lipid profiles by gender in the Taiwanese population. In our study, vegetarian diet lowered LDL-C and HDL-C levels in men. HDL-C and HDL-C:TC were equally lowered in women, whereas TAG level was increased. Vegetarian diets were associated with higher CVD risk in women than men.

From the survey, the percentage of male vegans was $1.3 \%$ while female vegans constituted $2.8 \%$. The traditional Taiwanese diet contains plenty of plant-based foods, fish in low to moderate amounts, poultry and eggs used in low amounts, red meat used sparingly and the absence of dairy products ${ }^{(17)}$. The tofu and other soyabean products, wheat protein, beans, nuts, pickles and plain rice in Taiwanese folk vegetarian meals are normally considered as a 'light' type of diet with less amounts of fat ${ }^{(18)}$. From a national survey, elderly vegetarians in Taiwan were noted to have significantly lower daily total energy intake, lower TC, higher percentage of PUFA, higher $\mathrm{Ca}$ and higher crude fibre intakes compared with omnivores ${ }^{(19)}$.

However, a study conducted in a private membership chain clinic in Taiwan showed that veganism increased the risk of metabolic syndrome, high TAG and low HDL-C ${ }^{(20)}$. From a hospital-based study, healthy male vegetarians were noted with low TC and LDL-C while healthy female vegetarians had low TC levels ${ }^{(8)}$. In addition to lower TC, LDL-C and HDL-C, Taiwanese female vegetarians had higher levels of high-sensitivity C-reactive protein, homocysteine and TAG $^{(21)}$. Besides, female Buddhists had significantly lower TC:HDL-C and LDL-C:HDL-C ratios ${ }^{(9)}$. Postmenopausal vegetarians had lower TC, LDL-C and HDL-C compared with age-matched omnivores ${ }^{(22)}$. In the present study, female vegetarians had lower HDL-C and higher levels of TAG compared with omnivores, whereas Rizzo et al. reported higher HDL-C and lower TAG concentrations in vegetarians $^{(23)}$. Reductions in the intake of dietary fats are associated with lower plasma LDL-C and HDL-C levels ${ }^{(12)}$. Healthy untrained individuals consuming a low-fat diet were found to have lower HDL-C ${ }^{(24)}$. Vegetarians in Taiwan had lower fat intake with low serum TC and TAG levels compared with omnivores ${ }^{(25)}$. In our study, vegan diet contributed to low HDL-C levels in both genders. There were sex disparities in the LDL-C, TAG and HDL-C:TC levels among diet patterns. However, the cross-sectional design was inadequate to explain such disparities. 
Vegetarian diets were associated with lower HDL-C:TC in women after adjusting for confounders. The ratio TC:HDL-C (the reverse of HDL-C:TC) has been reported to be a better index for CVD than TC, HDL-C or LDL-C ${ }^{(6,26)}$, or even LDL-C:HDL-C ${ }^{(27)}$. Lack of all animal products in a diet increases the risk of certain nutritional deficiencies, including those of vitamins $\mathrm{B}_{12}$ and $\mathrm{D}$, Ca and long-chain $n-3$ fatty acids $^{(1)}$. Because of a substantial portion of essential nutrients in dairy products, especially $\mathrm{Ca}, \mathrm{K}$ and $\mathrm{Mg}$, the ovo-lacto vegetarian diet is beneficial for reducing the risk of metabolic syndrome, stroke and some cancers $^{(28)}$. Our results indicated that the effects of vegetarian and ovo-lacto diets on lipid profiles were similar among women, unlike men.

Central obesity, low HDL-C and high TAG have been considered as components of the metabolic syndrome ${ }^{(29)}$ and independent risk factors for $\mathrm{CVD}^{(30,31)}$. Measures of abdominal obesity, principally waist circumference and WHR, are used as surrogates of body fat centralization and have been used to evaluate CVD risk $^{(31,32)}$. Obesity was more strongly related to TAG than other variables in both sexes. Some important confounding factors such as exercise can contribute in increasing HDL-C:TC ${ }^{(33)}$.

Consumers purchase organic foods for different reasons some of which include concerns about pesticide and antibiotic residues, human health and delicacy. In a systematic review, biomarkers and nutrient levels in serum, urine, breast milk and semen in adults did not show any clinically meaningful differences between conventional and organic foods. However, residues of pesticides and antibiotic-resistant bacteria were present in small amounts $^{(34)}$. In the present study organic food did not have any significant effect on lipid profiles.

There were several limitations in the present study. First, TwSHHH was a cross-sectional study that was susceptible to unmeasured confounders and reverse causation. Second, a potential self-selection effect may have existed among persons who adopted vegetarian diets because they may have been more aware of health concerns. We stratified the participants by sex, age, organic food consumption and exercise. Participants who received lipid-lowering therapy were excluded. Third, the questionnaires did not contain detailed information on the dietary contents to clarify whether it was a diet with mostly polyunsaturated rather than monounsaturated fat. This made it harder to clarify the relationship between cardiometabolic profiles. Finally, the small sample size of the vegans and ovo-lacto vegetarians may not have been sufficient to detect the difference between vegetarians and omnivores. Multivariate regression was used to evaluate the relationship between lipid profiles and explanatory variables, including age, WHR, menopause and other variables. We did not analyse the data by the matching method. Even with careful matching of the pairs, there would still be many other factors influencing lipid profiles which may be impossible to match.

\section{Conclusions}

Vegetarian diet resulted in a significant decrease in HDL-C:TC and a significant increase in TAG levels in females, as well as a significant decrease in HDL-C concentrations in both males and females. Ovo-lacto vegetarian diet may be more appropriate for men. The present results cannot directly address biological and nutritional mechanisms underlying the findings; further studies are needed to investigate such mechanisms.

\section{Acknowledgements}

Acknowledgments: This study is based on the data obtained from the 2002 Taiwanese Survey on Hypertension, Hyperglycemia, and Hyperlipidemia (TwSHHH), provided by the Bureau of Health Promotion, Department of Health, Taiwan. The descriptions or conclusions herein do not represent the viewpoints of the Bureau. Financial support: This research received no specific grant from any funding agency in the public, commercial or not-for-profit sectors. Conflict of interest: None. Authorship: Y.-P.L. conceived and designed the study. C.-C.L., P.-C.K. and C.-C.H. analysed and interpreted the data. Z.-H.J., H.-C.C., Y.-C.C., Y.-C. Liaw and Y.-C. Liang drafted the manuscript. Z.-H.J. and O.N.N. did a critical revision of the manuscript. All authors contributed to, read and approved the final version of the manuscript. Etbics of human subject participation: Ethical approval was not required.

\section{References}

1. Craig WJ (2009) Health effects of vegan diets. Am J Clin Nutr 89, issue 5, 1627S-1633S.

2. Key TJ, Fraser GE, Thorogood M et al. (1999) Mortality in vegetarians and nonvegetarians: detailed findings from a collaborative analysis of 5 prospective studies. Am J Clin Nutr 70, 3 Suppl., 516S-524S.

3. Fraser GE (2009) Vegetarian diets: what do we know of their effects on common chronic diseases? Am J Clin Nutr 89, issue 5, 1607S-1612S.

4. McEvoy CT, Temple N \& Woodside JV (2012) Vegetarian diets, low-meat diets and health: a review. Public Health Nutr 15, 2287-2294.

5. Pettersen BJ, Anousheh R, Fan J et al. (2012) Vegetarian diets and blood pressure among white subjects: results from the Adventist Health Study-2 (AHS-2). Public Health Nutr 15, 1909-1916.

6. Ridker PM, Rifai N, Cook NR et al. (2005) Non-HDL cholesterol, apolipoproteins A-I and B100, standard lipid measures, lipid ratios, and CRP as risk factors for cardiovascular disease in women. JAMA 294, 326-333.

7. Anon. (2001) Executive Summary of The Third Report of The National Cholesterol Education Program (NCEP) Expert Panel on Detection, Evaluation, and Treatment of High Blood Cholesterol in Adults (Adult Treatment Panel III). JAMA 285, 2486-2497.

8. Chen CW, Lin YL, Lin TK et al. (2008) Total cardiovascular risk profile of Taiwanese vegetarians. Eur J Clin Nutr 62, 138-144. 
9. Chiang JK, Lin YL, Chen CL et al. (2013) Reduced risk for metabolic syndrome and insulin resistance associated with ovo-lacto-vegetarian behavior in female Buddhists: a case-control study. PloS one $\mathbf{8}$, e71799.

10. Fu CH, Yang CC, Lin CL et al. (2008) Alteration of cardiovascular autonomic functions by vegetarian diets in postmenopausal women is related to LDL cholesterol levels. Chin J Physiol 51, 100-105.

11. Zhang Z, Ma G, Chen S et al. (2013) Comparison of plasma triacylglycerol levels in vegetarians and omnivores: a metaanalysis. Nutrition 29, 426-430.

12. Ashen MD \& Blumenthal RS (2005) Clinical practice. Low HDL cholesterol levels. $N$ Engl J Med 353, 1252-1260.

13. Huang YC, Ho CC, Lin PT et al. (2010) Optimal cutoff value of high-density lipoprotein cholesterol for predicting coronary artery disease in Taiwanese population. Nutr Res 30, 21-26.

14. Villablanca AC, Jayachandran M \& Banka C (2010) Atherosclerosis and sex hormones: current concepts. Clin Sci (Lond) 119, 493-513.

15. World Health Organization (2008) Waist Circumference and Waist-Hip Ratio: Report of a WHO Expert Consultation. WHO: Geneva.

16. De Biase SG, Fernandes SF, Gianini RJ et al. (2007) Vegetarian diet and cholesterol and triglycerides levels. Arq Bras Cardiol 88, 35-39.

17. Lin YC, Yen LL, Chen SY et al. (2003) Prevalence of overweight and obesity and its associated factors: findings from National Nutrition and Health Survey in Taiwan, 1993-1996. Prev Med 37, 233-241.

18. Chen YC, Chiang CI, Lin RS et al. (2005) Diet, vegetarian food and prostate carcinoma among men in Taiwan. $\mathrm{Br} \mathrm{J}$ Cancer 93, 1057-1061.

19. Huang CJ, Fan YC, Liu JF et al. (2011) Characteristics and nutrient intake of Taiwanese elderly vegetarians: evidence from a national survey. Br J Nutr 106, 451-460.

20. Shang P, Shu Z, Wang Y et al. (2011) Veganism does not reduce the risk of the metabolic syndrome in a Taiwanese cohort. Asia Pac J Clin Nutr 20, 404-410.

21. Chen CW, Lin CT, Lin YL et al. (2011) Taiwanese female vegetarians have lower lipoprotein-associated phospholipase A2 compared with omnivores. Yonsei Med J 52, 13-19.
22. Su TC, Torng PL, Jeng JS et al. (2011) Arterial function of carotid and brachial arteries in postmenopausal vegetarians. Vasc Health Risk Manag 7, 517-523.

23. Rizzo NS, Sabate J, Jaceldo-Siegl K et al. (2011) Vegetarian dietary patterns are associated with a lower risk of metabolic syndrome: the Adventist Health Study 2. Diabetes Care 34, 1225-1227.

24. Meksawan K, Pendergast DR, Leddy JJ et al. (2004) Effect of low and high fat diets on nutrient intakes and selected cardiovascular risk factors in sedentary men and women. J Am Coll Nutr 23, 131-140.

25. Yen CE, Yen CH, Huang MC et al. (2008) Dietary intake and nutritional status of vegetarian and omnivorous preschool children and their parents in Taiwan. Nutr Res 28, 430-436.

26. Jiang R, Schulze MB, Li T et al. (2004) Non-HDL cholesterol and apolipoprotein B predict cardiovascular disease events among men with type 2 diabetes. Diabetes Care 27, 1991-1997.

27. Ballantyne CM \& Hoogeveen RC (2003) Role of lipid and lipoprotein profiles in risk assessment and therapy. $A m$ Heart J 146, 227-233.

28. Weaver CM (2009) Should dairy be recommended as part of a healthy vegetarian diet? Point. Am J Clin Nutr 89, issue 5, 1634S-1637S.

29. Alberti KG, Zimmet P \& Shaw J (2005) The metabolic syndrome - a new worldwide definition. Lancet $\mathbf{3 6 6}$, 1059-1062.

30. Toth PP (2005) High-density lipoprotein as a therapeutic target: clinical evidence and treatment strategies. $\mathrm{Am} \mathrm{J}$ Cardiol 96, 50K-58K.

31. Schneider HJ, Friedrich N, Klotsche J et al. (2010) The predictive value of different measures of obesity for incident cardiovascular events and mortality. J Clin Endocrinol Metab 95, 1777-1785.

32. Schneider HJ, Glaesmer H, Klotsche J et al. (2007) Accuracy of anthropometric indicators of obesity to predict cardiovascular risk. J Clin Endocrinol Metab 92, 589-594.

33. Tseng ML, Ho CC, Chen SC et al. (2013) A simple method for increasing levels of high-density lipoprotein cholesterol: a pilot study of combination aerobic- and resistanceexercise training. Int J Sport Nutr Exerc Metab 23, 271-281.

34. Smith-Spangler C, Brandeau ML, Hunter GE et al. (2012) Are organic foods safer or healthier than conventional alternatives?: a systematic review. Ann Intern Med 157, 348-366. 